\title{
Diagnosis of Colorectal Tumor Invasion by Endoscopic Miniature Probe Ultrasonography
}

\author{
SHIRO MIYAZAKI \\ The Second Department of Medicine, Kurume University School of Medicine, \\ Kurume 830-0011, Japan
}

\begin{abstract}
Summary: We evaluated the diagnostic efficacy of high-frequency $(20 \mathrm{MHz})$ ultrasound probe (HFUP) for the staging of invasive depth of colorectal tumors. The subjects were 27 patients with colorectal tumorous lesions who were treated by endoscopic or surgical operation (11 patients with lesions remaining in the mucosa, 13 patients with submucosal cancer, and 3 patients with cancer invading the muscularis propria or deeper layers). Considering the previous reports that endoscopic mucosal resection (EMR) is indicated in cases of tumors remaining in the mucosa or cancer slightly invading the submucosal layer, we divided the submucosal layer vertically into three equal areas (most superficial, middle and deepest areas which were referred to as $\mathrm{sm} 1, \mathrm{sm} 2$ and $\mathrm{sm} 3$, respectively). The depth of tumor invasion in histological specimens was compared to the depth of tumor invasion as assessed preoperatively using the HFUP. The HFUP-based diagnosis was identical to the histological diagnosis in $86.4 \%$ of all cases, when the depth of tumor invasion was rated on a three-point scale: (i) $\mathrm{m}$-sm1, (ii) sm2-sm3 or (iii) $\mathrm{mp}$ or deeper. These results indicate that the HFUP is very useful in selecting a therapeutic method for colorectal tumors.
\end{abstract}

Key words colorectal tumor, endoscopic ultrasonography, miniature probe, diagnosis of tumor invasion

\section{INTRODUCTION}

In recent years, the superficial colorectal tumors have been frequently detected at many medical facilities [1-3], and endoscopic mucosal resection (EMR) has often been used in these cases [1,4]. The selection of a therapeutic method for early colorectal cancer, especially determination of the indication of endoscopic treatment for this cancer, is a clinically important. Cases of superficial colorectal cancer without lymph node metastasis are absolutely indicated for endoscopic treatment. Cancers remaining in the mucosa are usually free of lymph node metastasis and can be regarded as being indicated for endoscopic treatment. Endoscopic treatment of submucosal cancer is reported to involve several problems. Submucosal (sm) cancers are equally subdivided vertically into $\mathrm{sm} 1, \mathrm{sm} 2$ and $\mathrm{sm} 3$, depending on whether or not cancer has invaded the most superficial $(\mathrm{sm} 1)$, middle $(\mathrm{sm} 2)$ or deepest $(\mathrm{sm} 3)$ layer of the submucosa. Cancers invading the sm1 or less deeply are considered to be indicated for endoscopic treatment, because lymph node metastasis is rare in these cases. Cancers invading the $\mathrm{sm} 2$ or $\mathrm{sm} 3$ involve higher probability of lymph node metastasis and some probability of distant metastasis, and are therefore considered to be indicated for radical operation, combined with lymph node excision. As to the cancer invading the muscularis propria or more deeper, it is considered to be indicated for more wide area excision of lymph node than $\mathrm{sm} 2$ or $\mathrm{sm} 3$ cancer. To select the optimum therapeutic method for a given case, it is necessary to accurately assess the depth of tumor invasion on a three-point scale: (i) lesions remaining in the mucosa (m) or sm1, (ii) lesions reaching the $\mathrm{sm} 2$ or $\mathrm{sm} 3$, and (iii) lesions invading the muscularis propria $(\mathrm{mp})$ or more deeply.

$\mathrm{X}$-rays and endoscopy have conventionally been used to assess the depth of tumor invasion. Following the recent development of a high-frequen- 
cy small-diameter ultrasound probe (HFUP), the usefulness of this probe in assessing the depth of colorectal tumor invasion has begun to be reported [5-10].

The present study was undertaken to assess the usefulness of endoscopic ultrasonography using an HFUP $(20 \mathrm{MHz})$ in assessing the depth of invasion of colorectal tumorous lesions.

\section{MATERIALS AND METHODS}

The subjects were 27 patients with colorectal tumorous lesions who had undergone endoscopic ultrasonography and subsequent endoscopic or surgical operation at the Second Department of Internal Medicine or the Division of Gastroenterology of the Kurume University Hospital between July 1994 and December 1995 and in whom the resected tissue was examined histopathologically. There were 5 cases of adenoma, 6 cases of $\mathrm{m}$ cancer, 13 cases of sm cancer, and 3 cases of advanced cancer. The shape of lesions was macroscopically classified into the following types according to the macroscopic classification system established by the Japanese Research Society for Cancer of the Colon and Rectum: type I (protruding type), type II (superficial type), noduleaggregating type and advanced type. Type I was subdivided into type Ip (pedunculated), Isp (subpedunculated) and Is (sessile). Type II was subdivided into IIa (superficial elevated type), IIb (superficial flat type) and IIc (superficial depressed type). One lesion was rated as type Ip, one lesion as type Isp, one lesion as type Is, 11 lesions as type IIa, no lesion as type IIb, 8 lesions as type IIc, 2 lesions as nodule-aggregating type and 3 lesions as advanced type (Table 1).

A high-frequency ultrasound miniature probe (sp501, 20 MHz, Fuji Corp, Tokyo, Japan) was used for endoscopic ultrasonography by the degassed water filling method.

The normal colonic wall has a five-layer structure. The first and second layers were the mucosal layers. The third layer corresponded to the submucosal layer. The fourth layer was the muscularis propria. The fifth layer was the serosa. The submucosal layer (sm) was equally divided vertically into three areas, i.e., the most superficial (SM1), middle (SM2) and deepest (SM3) areas. The histological depth of tumor invasion of the submucosal layer was rated on three areas according to the classification of Kudo et al. [11] : sm1, sm2 and sm3.

The ability of endoscopic ultrasonography to provide images of the structure of tumorous lesions was analyzed in relation to the height of lesions. Cases in which the five-layer structure of the colorectal wall adjacent to the lesion was visible were classified as cases in which lesions could be visualized. Cases in which lesions and the five-layer structure of the adjacent area were not visible were classified as cases in which lesions could not be visualized. The height of lesions, i.e., the distance from the surface of normal marginal mucosa to the top of the lesion, was measured under a microscope.

In cases where lesions were clearly visible, the

TABLE 1.

Macroscopic classification and histologic depth of invasion

\begin{tabular}{|c|c|c|c|c|c|c|}
\hline \multirow{2}{*}{ Gross appearance } & \multicolumn{5}{|c|}{ Histology } & \multirow{2}{*}{ Total } \\
\hline & $\mathrm{m}^{*}$ & sm1 & $\mathrm{sm} 2$ & $\mathrm{sm} 3$ & $\mathrm{mp}$ & \\
\hline Protruding type (I) & 1 & & (1) & (1) & & $1(2)$ \\
\hline Superficial elevated type (IIa) & $7(2)$ & & 2 & & & $9(2)$ \\
\hline Superficial depressed type (IIc) & & 2 & 4 & 2 & & 8 \\
\hline Nodule-aggregating lesion & 1 & & (1) & & & $1(1)$ \\
\hline Borrman 2 & & & & & 3 & 3 \\
\hline Total & $9(2)$ & 2 & $6(2)$ & $2(1)$ & 3 & $22(5)$ \\
\hline
\end{tabular}

m: mucosal cancer; sm: submucosal cancer; mp: cancer extending into the muscularis propria

*Includes adenoma $\quad($ ): invisible lesions 
depth of tumor invasion as assessed by endoscopic ultrasonography was compared with the histological depth of invasion, and the coincidence rate was calculated. Furthermore the coincidence rate for the selection of therapeutic methods was analyzed by dividing the lesions into three groups: (i) $\mathrm{m}$ or $\mathrm{sm} 1$ lesions, (ii) $\mathrm{sm} 2$ or $\mathrm{sm} 3$ lesions and (iii) $\mathrm{mp}$ or deeper lesions. When the depth of tumor invasion was determined by endoscopic ultrasonography, the presence or absence of destruction of the five-layer structure due to tumor-associated low echoes was checked for, in accordance with the method reported by Aibe et al. [12]. Cases in which low echoes were visible in the first and second layers but were absent in the third and subsequent layers were rated as having $\mathrm{m}$ lesions. Cases in which low echoes were visible in the third layer but absent in the fourth and deeper layers were rated as having sm lesions.

The ability of endoscopic ultrasonography to allow accurate diagnosis of the depth of tumor invasion (the accurate diagnosis rate) was analyzed, by comparing the depth of tumor invasion as determined by endoscopic ultrasonography with the depth as determined histopathologically for tumors classified by the maximum diameter into four groups: 6-10 $\mathrm{mm}, 11-15 \mathrm{~mm}, 16-20 \mathrm{~mm}$ and over $21 \mathrm{~mm}$. Furthermore, the accurate diagnosis rate was analyzed for three categories of tumor depth (m-sm1, sm2-3 and mp or deeper).

\section{RESULTS}

The ability to obtain images of the five-layer structure of the colonic wall and the tumor height (Table 2)
The five-layer structure of lesions was visible in $22(81.5 \%)$ of the 27 cases but not in $5(18.5 \%)$ when examined by endoscopic ultrasonography. The tumor height in cases where the structure was not visible $(5.73 \pm 4.44 \mathrm{~mm})$ was greater than that in cases where it was visible $(3.16 \pm 2.52 \mathrm{~mm})$, although the difference was not significant.

The relationship between accurate diagnosis rate and the depth of tumor invasion

For the 22 lesions which were depicted well by endoscopic ultrasonography, the accurate diagnosis rate was analyzed in relation to the depth of tumor invasion assessed histopathologically.

Accurate diagnosis rate in relation to the depth of tumor invasion (Table 3): The accurate diagnosis rate was $63.6 \%(14 / 22)$ for all patients, $44.4 \%$ (4/9) for $\mathrm{m}$ lesions, $50.0 \%(1 / 2)$ or sm1 lesions, $83.3 \%(5 / 6)$ for sm2 lesions, $50.0 \%(1 / 2)$ for sm3 lesions, and $100.0 \%$

TABLE 2.

The ability to obtain images of the five-layer structure of the colonic wall and tumor height

\begin{tabular}{cc}
\hline & Height of lesions $(\mathrm{mm})$ \\
\hline $\begin{array}{c}\text { Invisible lesions } \\
(\mathrm{n}=5)\end{array}$ & $5.73 \pm 4.44$ \\
$\begin{array}{c}\text { Visible lesions } \\
(\mathrm{n}=22)\end{array}$ & $3.16 \pm 2.52$ \\
\hline
\end{tabular}

TABLE 3.

Accurate diagnosis rate in relation to the depth of tumor invasion

\begin{tabular}{|c|c|c|c|c|c|c|c|}
\hline \multirow{2}{*}{ Histology } & \multicolumn{5}{|c|}{ HFUP } & \multirow{2}{*}{ Total } & \multirow{2}{*}{ Accuracy $(\%)$} \\
\hline & $\mathrm{M}^{*}$ & SM1 & SM2 & SM3 & MP & & \\
\hline $\mathrm{m}^{*}$ & 4 & 3 & & 2 & & 9 & $4 / 9(44.4)$ \\
\hline $\operatorname{sm} 1$ & & 1 & 1 & & & 2 & $1 / 2(50.0)$ \\
\hline $\operatorname{sm} 2$ & & & 5 & 1 & & 6 & $5 / 6(83.3)$ \\
\hline $\operatorname{sm} 3$ & & & 1 & 1 & & 2 & $1 / 2(50.0)$ \\
\hline $\mathrm{mp}$ & & & & & 3 & 3 & $3 / 3(100)$ \\
\hline Total & 4 & 4 & 7 & 4 & 3 & 22 & $14 / 22(63.6)$ \\
\hline
\end{tabular}

*Includes adenoma HFUP: high-frequency ultrasound probe 
TABLE 4.

Accurate diagnosis rate in relation to the depth of tumor invasion clinically used for the selection of therapeutic methods

\begin{tabular}{lccccc}
\hline \multirow{2}{*}{ Histology } & \multicolumn{3}{c}{ HFUP } & Total & $\begin{array}{c}\text { Accuracy } \\
(\%)\end{array}$ \\
\cline { 2 - 5 } $\mathrm{m}^{*}-\mathrm{sm} 1$ & $\mathrm{M}^{*}-\mathrm{SM} 1$ & $\mathrm{SM} 2-\mathrm{SM} 3$ & MP - & 11 & $8 / 11(72.7)$ \\
$\mathrm{sm} 2-\mathrm{sm} 3$ & 8 & 3 & & 8 & $8 / 8(100)$ \\
$\mathrm{mp}-$ & 8 & 3 & 3 & $3 / 3(100)$ \\
\hline Total & 8 & 11 & 3 & 22 & $19 / 22(86.4)$ \\
\hline
\end{tabular}

*Includes adenoma HFUP: high-frequency ultrasound probe

(3/3) for mp lesions. In 5 cases where histological diagnosis was mucosal tumor, the evaluation of the depth of tumor invasion by endoscopic ultrasonography were $\mathrm{sm} 1$ or sm3. Thus, the depth of tumor invasion tended to be overestimated in cases of mucosal lesions.

Accurate diagnosis rate in relation to three categories of tumor invasion depth clinically used for the selection of therapeutic methods (Table 4): When the accurate diagnosis rate was analyzed in relation to three categories of tumor invasion depth ( $\mathrm{m}$-sml, sm2-3 and $\mathrm{mp}$ or deeper), the accurate diagnosis rate was $86.4 \%(19 / 22)$ for all patients, $72.7 \%(8 / 11)$ for m-sm1 cases, $100.0 \%(8 / 8)$ for $\mathrm{sm} 2-3$ cases and $100.0 \%(3 / 3)$ for $\mathrm{mp}$ or deeper cases. Thus, the accurate diagnosis rate was high for all three categories.

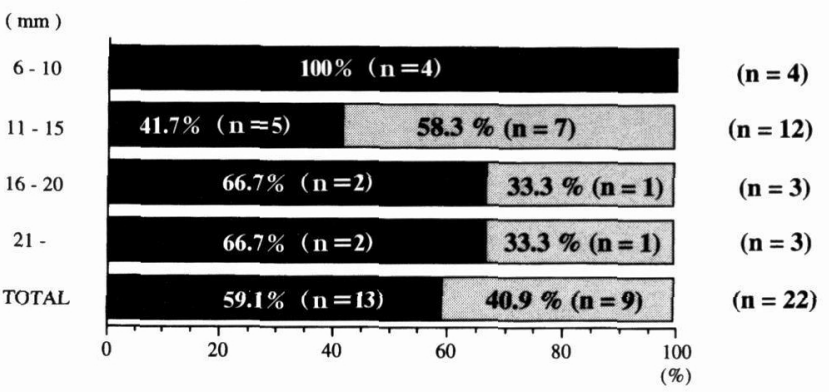

Fig. 1. Relationship between the tumor diameter and accurate diagnosis of the depth of tumor invasion. Five categories of the depth of tumor invasion (m, sm1, sm2, $\mathrm{sm} 3$ and $\mathrm{mp}$ or deeper) are used in evaluating the invasive degree. Closed squares indicate the accurate diagnosis rate. Open squares indicate the erroneous diagnosis rate.
Assessment of the depth of tumor invasion in relation to the tumor size

Tumor size and the accuracy in assessing the depth of tumor invasion (Fig. 1): The percentage of cases in which the depth of tumor invasion was assessed accurately was $100.0 \%$ (4.4) when the tumor diameter was $6-10 \mathrm{~mm}, 41.7 \%(5 / 12)$ at a tumor diameter of $16-20 \mathrm{~mm}$, and $66.7 \%(2 / 3)$ at a tumor diameter over $21 \mathrm{~mm}$. Thus, the percentage did not correlate with the tumor diameter.

Relationship between tumor diameter and accurate diagnosis rate using three categories of tumor depth (Fig. 2): When the percentage of cases in which tumor invasion depth (m-sm1, sm2-3 and $\mathrm{mp}$ or deeper) was assessed accurately was analyzed in relation to tumor diameter, the percentage was $100.0 \%(4 / 4)$ when the tumor diameter was 6-10

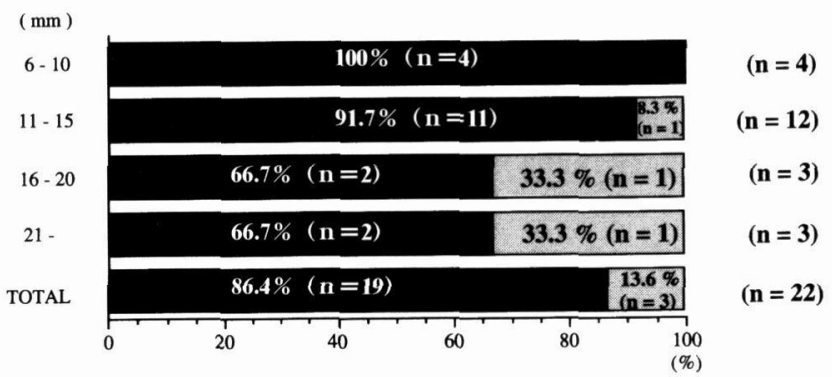

Fig. 2. Relationship between tumor diameter and tumor depth assessment using three categories of tumor depth. Three categories of the depth of tumor invasion ( $\mathrm{m}-\mathrm{sm} 1$, sm2-3 and $\mathrm{mp}$ or deeper) are used in evaluating the invasive degree. Closed squares indicate the accurate diagnosis rate. Open squares indicate the erroneous diagnosis rate 
$\mathrm{mm}, 91.7 \%(11 / 12)$ at a tumor diameter of $11-15$ $\mathrm{mm}, 66.7 \%(2 / 3)$ at a tumor diameter of $16-20 \mathrm{~mm}$ and $66.7 \%$ at a tumor diameter over $21 \mathrm{~mm}$. The percentage tended to be higher in smaller tumors than in larger tumors, although the difference was not significant. Thus, no significant correlation was observed between the tumor diameter and the accurate assessment of tumor invasion depth using the three-category classification of tumor depth.

\section{Case illustration}

Firstly, a representative case of accurate diagnosis evaluated using endoscopic ultrasonography is described. Colonoscopy detected a superficial elevated tumor with central depression in the sigmoid colon (Fig. 3A). Use of the dye-spraying technique more clearly delineated the lesion (Fig. 3B). Use of the HFUP showed that the lesion had invaded the middle of the third layer (Fig. 3C). Histologically, the section shows that the lesion involved two-thirds of the submucosal layer (Fig. 3D). Secondly, a representative case of the erroneous diagnosis evaluated using endoscopic ultrasonography is described. Colonoscopy detected a superficial depressed tumor with a central nodule in the transverse colon using dye-spraying technique (Fig. 4A). Use of the HFUP showed that the lesion had invaded two-thirds of the third layer (Fig. 4B). Histology revealed that the lesion had involved one-third of submucosal layer. The lesion of high magnification showed the abundant fibrosis under the nodule (Fig. 4C, D).
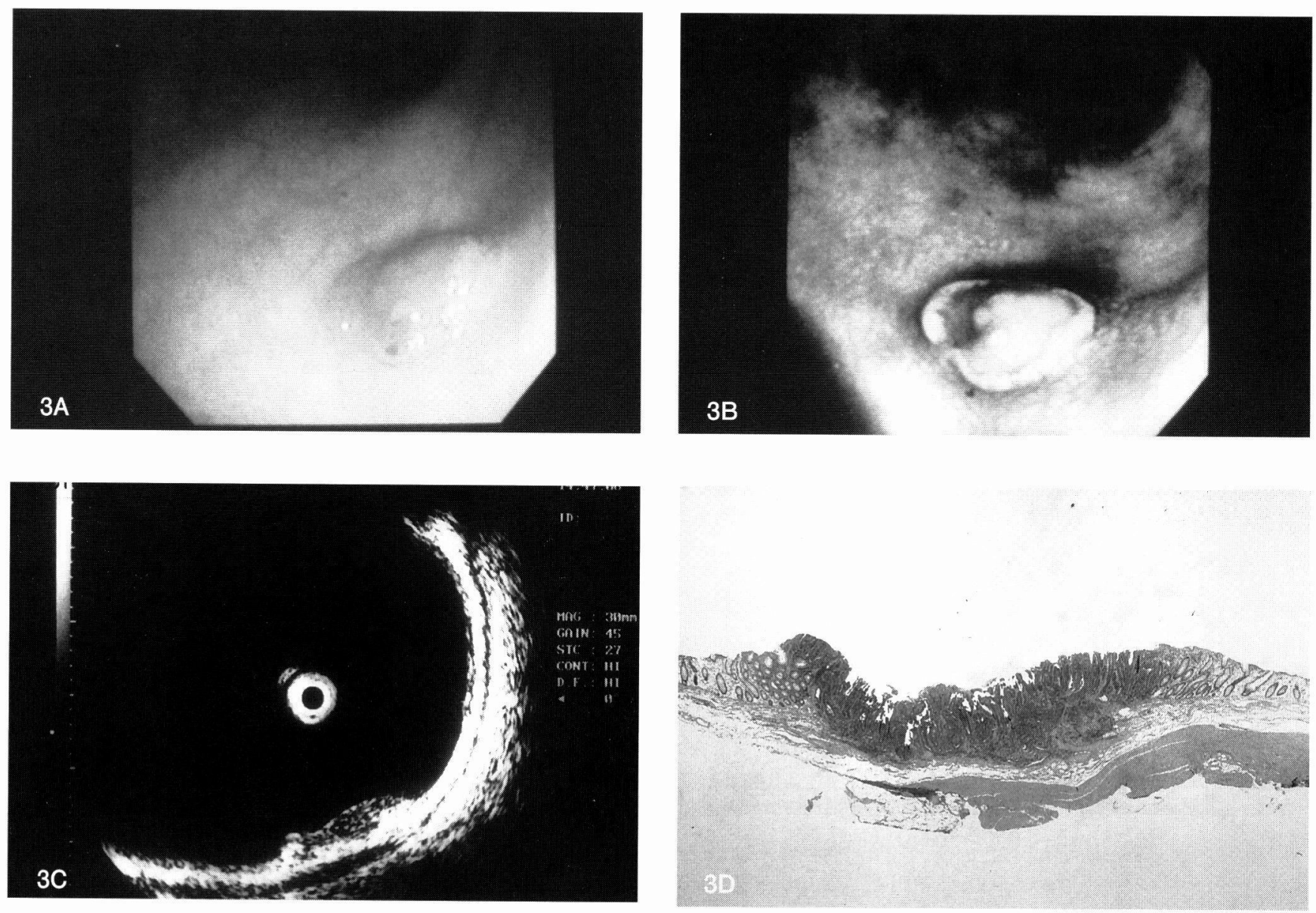

Fig. 3. A case of accurate diagnosis. A: Colonoscopy revealed a reddish superficial elevated tumor with central depression in the sigmoid colon. B: The dye-spraying technique demonstrates the lesion more clearly. C: HFUP showed that the lesion had invaded the middle of the third layer. (low echoic) (SM2) D: Histology revealed that the lesion involved two-thirds of the submucosal layer. ( $\mathrm{sm} 2)$ 

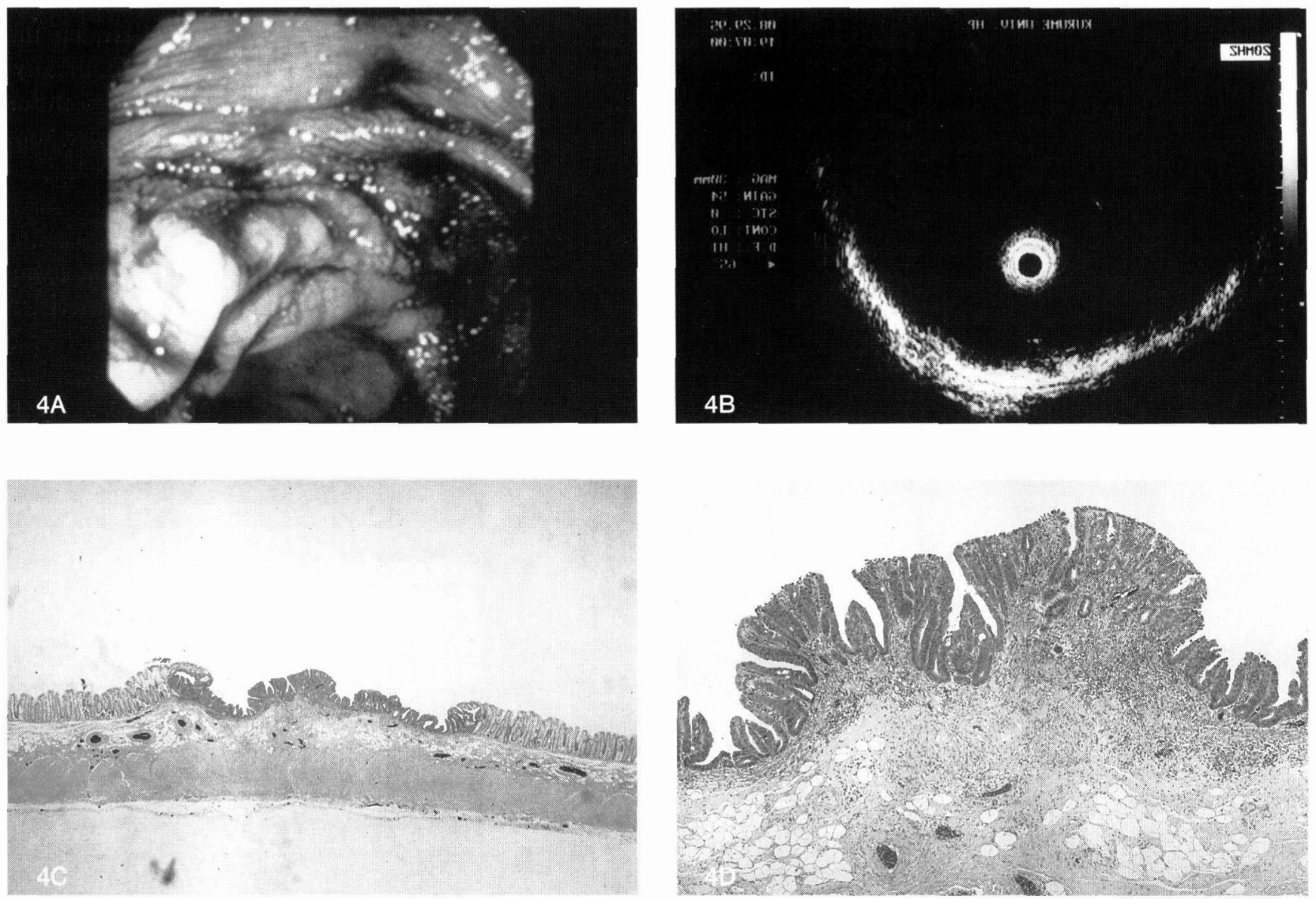

Fig. 4. A case of erroneous diagnosis. A: Colonoscopy revealed superficial depressed tumor in the transverse colon. B: HFUP showed that the lesion had invaded two-thirds of the third layer. (SM2) C: Histology revealed that the lesion had involved one-third of submucosal layer. $(\mathrm{sm} 1)$ D: Section showed the abundant fibrosis around the tumor frontier area in high magnification of lesion.

\section{DISCUSSION}

Due to the spread of colorectal examination, the number of colorectal cancer detected in early stages has been increasing. Radical treatment by polypectomy or EMR is possible for cancers remaining in the mucosa (m cancer). In cases of cancer which has invaded the submucosal layer (sm cancer), the indications for endoscopic treatment or surgical operation (combined with lymph node excision) need to be determined carefully, taking into account the possibility of residual cancer, lymph node metastasis and distant metastasis.

The depth of invasion by colorectal cancer has conventionally been assessed using X-rays and endoscopic findings [13-15]. These methods, however, do not allow direct assessment of deep invasion of tumors. Instead they only allow indirect assessment of the depth of tumor invasion on the basis of extent and height of lesions on X-rays and endoscopic findings. For more accurate assessment of the depth of tumor invasion, endoscopic ultrasonography, which provides direct images of tumor invasion in the vertical direction, has been increasingly used in addition to conventional methods. The usefulness of endoscopic ultrasonography has been suggested in recent reports [16-22].

At present, two types of endoscopic ultrasonography are used. One type is the conventional instrument composed of an ultrasound vibrator attached to the tip of an endoscope (EUS). The other is a highfrequency ultrasound miniature probe (HFUP) which is inserted through the forceps channel of an endoscope. The EUS uses either $7.5 \mathrm{MHz}$ or $12 \mathrm{MHz}$ frequency. Since ultrasound attenuation is small, the EUS is useful when diagnosing lesions with a great height or extra intestinal lesions. Lesions with small height such as superficial tumors are difficult to be 
detected by the EUS. Insertion of the EUS deeply into the large intestine requires considerably high skill [23]. The HFUP uses a fairly high frequency (20 $\mathrm{MHz}$ ). Since ultrasound attenuation is large, the HFUP is primarily used to evaluate superficial tumors. Since the HFUP is usually inserted through the forceps channel of a video endoscope, ultrasound images can be taken while monitoring the lesions endoscopically. Furthermore, it is easy to insert video endoscope deeply into large intestine.

In the present study, we assessed the usefulness of HFUP in diagnosing the depth of colorectal tumorous lesions. Regarding the relationship between the height of lesions and the ability to provide images of the layered structure of lesions, Ashiwara et al. [24] have reported that the accurate diagnosis rate was high for lesions below $7 \mathrm{~mm}$ in size, but that the rate was lower for lesions larger than $8 \mathrm{~mm}$. Matsunaga et al. $[25,26]$ also reported that $90-100 \%$ of superficial tumors were visible with this technique, but that only about $65 \%$ of lesions with large height were visible. In the present study, the height of lesions was $3.16 \pm 2.52 \mathrm{~mm}$ for lesions visible with this technique and $5.73 \pm 4.41 \mathrm{~mm}$ for lesions not visible, although the difference was not significant. This difference is probably because an increase in tumor height accelerates ultrasound attenuation and thus makes imaging of the deepest area of the tumor impossible.

Reports from other facilities showed that this technique allowed accurate assessment of the depth of tumor invasion in $80-94 \%$ of all cases, indicating the usefulness of this technique [5,24,26-28]. In the present study, the percentage of cases in which this technique allowed accurate assessment of the depth of tumor invasion was low $(63.3 \%, 14 / 22)$. However, when the depth of tumor invasion was divided into three categories ( $\mathrm{m}$-sm1, sm2-3 and $\mathrm{mp}$ or deeper), which are often used clinically when selecting therapeutic methods, the accurate diagnosis rate was higher, i.e., $86.4 \%(19 / 22)$ for the entire population, $72.7 \%(8 / 11)$ for $\mathrm{m}$-sm1 cases and $100 \%$ (11/11) for $\mathrm{sm} 2$ or deeper cases. Although the accurate diagnosis rate for $\mathrm{m}$-sml cases was not satisfactorily high, this will not cause any problem when selecting endoscopic treatment or surgical operation, since the depth of tumor invasion is usually assessed based on a general evaluation of $\mathrm{X}$ ray, endoscopic and ultrasound findings, instead of relying solely on ultrasound findings.

Few studies have examined the relationship between the width of lesions and the usefulness of endoscopic ultrasonography in accurate assessment of the depth of tumor invasion. Yoshimoto et al. [29] reported that the accurate diagnosis rate was $81 \%$ for lesions smaller than $30 \mathrm{~mm}$ and $14 \%$ for lesions over $30 \mathrm{~mm}$. In the present study, no particular relationship was observed between the tumor size and the accurate diagnosis rate. However, when the depth of tumor invasion was divided into three categories (m-sm1, sm2-3 and $\mathrm{mp}$ or deeper), the accurate diagnosis rate tended to decrease as the tumor diameter increased. This is probably because ultrasound scanning of the entire lesion is difficult for larger lesions. The depth of tumor invasion cannot be accurately assessed unless the deepest part of the tumor can be scanned. It is not easy to scan a large lesion completely, including its deepest portion. The small-diameter ultrasound probe used in the present study can be inserted through the forceps channel of an ordinary video endoscope. If the deepest area of the tumor identified by endoscopic observation is scanned with this probe, the accurate diagnosis rate will be improved.

In the 9 cases where the ultrasound diagnosis of the depth of tumor invasion was discrepant from the histological diagnosis, the discrepancy is probably because infiltration of inflammatory cells [30], fibrosis of the tumor frontier region [31] and the presence of lymph follicles caused overestimation of the depth of tumor invasion by endoscopic ultrasonography.

In the present study, a HFUP was used to assess the depth of invasion by colorectal tumorous lesions. This probe was found to have the following advantages. It was easier to insert and manipulate than the conventional probes used for endoscopic ultrasonography (EUS). The use of this probe allows ultrasound imaging under endoscopic guidance and subsequent efficient endoscopic treatment when needed. Ultrasonic checks for deep tumor invasion under endoscopic guidance is easier with this probe. A disadvantage with this probe is that in cases of tumors with large height, the ability to obtain images of minute invasion in the deep area is low. This problem needs to be resolved.

A HFUP was used to obtain images of 27 colorectal tumorous lesions. Its ability to provide images of these lesions and to accurately assess the depth of tumor invasion was examined. This probe was found to be very useful in obtaining images of superficial tumors with low height. Endoscopic ultrasonography using his probe is expected to provide a useful means of determining the indications of early colorectal cancer for endoscopic resection if its find- 
ings are combined with findings from conventional $\mathrm{X}$-ray and video endoscopy.

ACKNOWLEDGMENTS: The author thanks Prof. Kyuichi Tanikawa (The Second Department of Medicine, Kurume University School of Medicine), Prof. Atsushi Toyonaga (Division of Gatroenterology, Kurume University School of Medicine), Dr. Osamu Tsuruta and Dr. Mitsutake Fujita (The Second Department of Medicine, Kurume Unversity School of Medicine) for their clinical advice and suggestions.

\section{REFERENCES}

1. Kudo S. Endoscopic mucosal resection of flat and depressed types of early colorectal cancer. Endoscopy 1993; 25:455-461.

2. Tada S, Yao T, Iida M, Koga H, Hizawa K et al. A clinicopathologic study of small flat colorectal carcinoma. Cancer 1994; 74:2430-2435.

3. Shimoda T, Ikegami M, Fujisaki J, Matsui T, Aizawa S et al. Early colorectal carcinoma with special reference to its development de novo. Cancer 1989; 64:1138-1146.

4. Karita M, Tada M, Okita K, and Kodama T. Endoscopic therapy for early colon cancer: the strip biopsy resection technique. Gastrointest Endosc 1991; 37:128-132.

5. Saitoh Y, Obara T, Einami K, Nomura M, and Taruishi M. Efficacy of high-frequency ultrasound probes for the preoperative staging of invasion depth in flat and depressed colorectal tumors. Gastrointest Endosc 1996; 44:34-39.

6. Cho E, Ashihara T, Mizuma Y, Yasuda K, and Nakajima $M$. Use of an ultrasonic probe in the diagnosis of colorectal lesions: a comparative study with echocolonoscope [Abstract]. Gastrointest Endosc 1993; 39:A294.

7. Martin RW, Silverstein FE, and Kimmey MB. A 20$\mathrm{MHz}$ ultrasound system for imaging the intestinal wall. Ultrasound in Med Biol 1989; 15:273-280.

8. Kimmey MB, Martin RW, and Silverstein FE. Endoscopic ultrasound probes. Gastrointest Endosc 1990; 36:S40-S46.

9. Silverstein FE, Martin RW, Kimmey MB, Jiranek GC, Franklin DW et al. Experimental evaluation of an endoscopic ultrasound probe: in vitro and in vivo canine studies. Gastroenterology 1989; 96:1058-1062.

10. Kimmey MB, Martin RW, and Silverstein FE. Clinical application of linear ultrasound probes. Endoscopy 1992; 24 (Suppl 1):364-369.

11. Kudo S, Soga J, Yamamoto M, Koyama S, and Muto T. Treatment of colorectal sm-carcinomas. I To Chou (Stomach and Intestine) 1984; 19:1349-1356. (in Japanese)

12. Aibe T. A study on the structure of layers of the gastrointestinal wall visualized by means of the ultrasonic endoscope: the structure of layers of the esophageal wall and the colonic wall. Gastroenterol Endosc 1984; 26:1465-1473. (in Japanese)

13. Yokota T, Matsui T, Fukuda H, Yokoyama T, Oka M et al. Evaluation of the depth of early colorectal cancers by endoscopy. I To Chou (Stomach and Intestine) 1994; 29: 1261-1269. (in Japanese)
14. Hirakawa M, Fuchigami T, Iwashita A, Tai H, and Matsuzaka T. Diagnosis of the depth of invasion in early colorectal cancer: Differential diagnosis between $\mathrm{m}, \mathrm{sm} 1$ and sm2, 3 from the roentgenographic point of view. I To Chou (Stomach and Intestine) 1994; 29:1249-1259. (in Japanese)

15. Shimizu S, Tada M, Otsuka H, Iso A, Kitamura T et al. Evaluation of the depth of colorectal cancers using endoscopic ultrasonograpy in comparison with barium enema and colonoscopic examinations. I To Chou (Stomach and Intestine) 1993; 28:1183-1189. (in Japanese)

16. Roubein LD, David C, Dubrow R, Faintuch J, and Lynch P. Endoscopic ultrasonography in staging rectal cancer. Am J Gastroenterol 1990; 85:1391-1394.

17. Rösch T, Lorenz R, and Classen M. Endoscopic ultrasonography in the evaluation of colon and rectal disease. Gastrointest Endosc 1990; S33-S39.

18. Shimizu S, Tada M, and Kawai K. Use of endoscopic ultrasonography for the diagnosis of colorectal tumors. Endoscopy 1990; 22:31-34.

19. Gregory AB, Michael VS Jr, Ian CL, Victor WF, James $\mathrm{MC}$ et al. Endoscopic ultrasound in the pre-operative staging of rectal carcinoma. Gastrointest Endosc 1992; 38:468-471.

20. Cho E, Nakajima M, Yasuda K, Ashihara T, and Kawai $\mathrm{K}$. Endoscopic ultrasonography in the diagnosis of colorectal cancer invasion. Gastrointest Endosc 1993; 39:521-527.

21. Rösch T. Endoscopic ultrasonography. Endoscopy 1994; 26:148-168.

22. xHulsmans FJH, Tio TL, Fockens P, Bosma A, and Tytgat GNJ. Assessment of tumor infiltration depth in rectal cancer with transrectal sonography: caution is necessary. Radiology 1994; 190:715-720.

23. Oohara N. A study on wall structure of the colon and early superficial cancer by $20 \mathrm{MHz}$ ultrasonography. Gastroenterol Endosc 1992; 34:2527-2539.

24. Ashihara T, Cho E, Nakajima M, Yasuda K, Mukai H et al. Diagnosis of early colorectal tumor invasion by endoscopic ultrasonography with special reference to the detectability of degree of submucosal carcinomatous infiltration. Gastroenterol Endosc 1994; 36:1561-1567. (in Japanese)

25. Matsunaga A, Mochizuki F, Fujita N, Ando M, Tominaga $\mathrm{G}$ et al. Diagnosis of early colorectal cancer by endoscopic ultrasonography. Gastroenterol Endosc 1996; 38:279-287. (in Japanese)

26. Matsunaga A, Mochizuki F, Fujita N, Ando $M$, Tominaga $\mathrm{G}$ et al. Diagnosis of early colorectal cancer by microscanner: evaluation of degree of submucosal carcinomatous infiltration. Gastroenterol Endosc 1996; 38:1322-1331. (in Japanese)

27. Oohara N, Murata Y, Kawanami C, Sugiyama S, Tanaka $\mathrm{Y}$ et al. Ultrasonography in the colorectal lesion; a comparison between endoscopic ultrasonography $(7.5 \mathrm{MHz})$ and sonoprobe system $(20 \mathrm{MHz})$. Shoukaki Naishikyo No Shinpo (Progress of Gastrointestinal Endoscopy) 1991; 39:221-225. (in Japanese)

28. Shimizu S, Saito T, Yoshida K, Hirata M, Kitamura T et al. Diagnosis of the depth of early colorectal cancer by 
endoscopic ultrasonography based on the subdivision of degree of submucosal infiltration. I To Chou (Stomach and Intestine) 1994; 29:1271-1278. (in Japanese)

29. Yoshimoto K, Takada H, and Sakai Y. The evaluation and prospect on the endoscopic ultrasonography for colorectal cancers. Shoukaki Naishikyo No Shinpo (Progress of Gastrointestinal Endoscopy) 1991; 38:5458. (in Japanese)

30. Yamashita Y, Shirouzu K, Isomoto H, Morotomi T,
Kurohiji $\mathrm{T}$ et al. Evaluation of intrarectal ultrasonography for assessment of invasion of rectal cancer. Japanese Journal of Gastroenterology 1987; 84:868-877. (in Japanese)

31. Kobayashi K, Katsumata T, Naitou Y, Igarashi M, Saigenji K et al. Diagnosis of the depth of early colorectal cancer invasion by endoscopic ultrasonography. Shoukaki Naishikyo (Endoscopia Digestiva) 1992; 4:1345-1351. (in Japanese) 